\section{( OPEN ACCESS}

\title{
Systemic but not topical TRAIL-expressing mesenchymal stem cells reduce tumour growth in malignant mesothelioma
}

\author{
Elizabeth K Sage, ${ }^{1}$ Krishna K Kolluri, ${ }^{1}$ Katrina McNulty, ${ }^{1}$ Sofia Da Silva Lourenco, ${ }^{1}$ \\ Tammy L Kalber, ${ }^{1,2}$ Katherine L Ordidge, ${ }^{1,2}$ Derek Davies, ${ }^{3}$ Y C Gary Lee, ${ }^{4}$ \\ Adam Giangreco, ${ }^{1}$ Sam M Janes ${ }^{1}$
}

\begin{abstract}
- Additional material is published online only. To view please visit the journal online (http://dx.doi.org/10.1136/ thoraxjnl-2013-204110).

${ }^{1}$ Division of Medicine, Lungs for Living Research Centre, University College London, London, UK

${ }^{2}$ Division of Medicine and Institute of Child Health, UCL Centre of Advanced Biomedical Imaging, University College London, London, UK

${ }^{3}$ Flow Cytometry Laboratory, Cancer Research UK, London Research Institute, London, UK ${ }^{4}$ School of Medicine and Pharmacology, University of Western Australia, Sir Charles Gairdner Hospital, Perth, Australia
\end{abstract}

Correspondence to Dr Sam M Janes,

Lungs for Living Research Centre, UCL Respiratory,

Rayne Building, 5 University Street, London WC1E 6JF, UK; s.janes@ucl.ac.uk

Received 2 July 2013 Revised 27 October 2013 Accepted 24 January 2014 Published Online First 24 February 2014

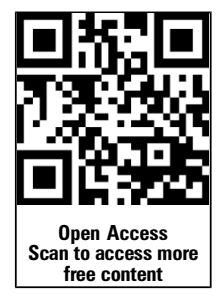

CrossMark

To cite: Sage EK, Kolluri KK, McNulty K, et al. Thorax 2014;69:638-647.

\begin{abstract}
Malignant pleural mesothelioma is a rare but devastating cancer of the pleural lining with no effective treatment. The tumour is often diffusely spread throughout the chest cavity, making surgical resection difficult, while systemic chemotherapy offers limited benefit. Bone marrow-derived mesenchymal stem cells (MSCs) home to and incorporate into tumour stroma, making them good candidates to deliver anticancer therapies. Tumour necrosis factor-related apoptosis-inducing ligand (TRAIL) is a pro-apoptotic molecule that selectively induces apoptosis in cancer cells, leaving healthy cells unaffected. We hypothesised that human MSCs expressing TRAIL (MSCTRAIL) would home to an in vivo model of malignant pleural mesothelioma and reduce tumour growth. Human MSCs transduced with a lentiviral vector encoding TRAIL were shown in vitro to kill multiple malignant mesothelioma cell lines as predicted by sensitivity to recombinant TRAIL (rTRAIL). In vivo MSC homing was delineated using dual fluorescence and bioluminescent imaging, and we observed that higher levels of MSC engraftment occur after intravenous delivery compared with intrapleural delivery of MSCs. Finally, we show that intravenous delivery of MSCTRAIL results in a reduction in malignant pleural mesothelioma tumour growth in vivo via an increase in tumour cell apoptosis.
\end{abstract}

\section{INTRODUCTION}

Malignant mesothelioma (MM) is a rare but devastating malignancy found most commonly within the pleura. It is largely caused by asbestos exposure ${ }^{1}$ and the mortality rate is increasing with $>2300$ deaths per year in the UK. ${ }^{2}$ Current treatment options are poor, and first-line chemotherapy with cisplatin and pemetrexed offers an average survival of 12 months. ${ }^{3}$ The role of radical surgery is controversial with the only large-scale clinical trial showing a trend to worse outcomes in patients undergoing extrapleural pneumonectomy. ${ }^{4}$ Because of the resistance of malignant pleural mesothelioma (MPM) to conventional treatments, new therapies are desperately needed. Most chemotherapy agents act by inducing tumour cell apoptosis via the intrinsic apoptotic pathway; however, MPM is known to be resistant to activation of this pathway, so interest has turned to activation of the extrinsic apoptotic pathway. ${ }^{5}$

\section{Key messages}

What is the key question?

- Can TRAIL delivered by mesenchymal stem cells (MSCs) be an effective therapeutic option in malignant pleural mesothelioma?

\section{What is the bottom line?}

- When delivered systemically, MSCs expressing TRAIL successfully incorporate into malignant pleural mesothelioma and induce cancer cell death.

\section{Why read on?}

- This is the first paper that uses both bioluminescent and fluorescent in vivo imaging to show MSC homing and infiltration into malignant pleural mesothelioma and delivery of TRAIL to cause a reduction in tumour burden.

Tumour necrosis factor (TNF)-related apoptosisinducing ligand (TRAIL) is a type II transmembrane protein and member of the TNF superfamily. It binds via two active transmembrane death receptors, DR4 and DR5, triggering the caspase cascade resulting in apoptosis. TRAIL is an exciting anticancer molecule as it induces cell death in cancer cells without affecting healthy cells. ${ }^{6}$ Phase I clinical trials looking at the use of both recombinant TRAIL (rTRAIL) ${ }^{7}$ and monoclonal antibodies to the TRAIL death receptors, DR4 and DR5, have shown promising results. ${ }^{8} 9$ However, there are problems with both of these treatment options. The half-life of rTRAIL is short at $32 \mathrm{~min}$, meaning multiple infusions are needed to deliver therapeutic dose systemic therapy. ${ }^{6}$ While monoclonal antibodies have the advantage of a receptor-specific high-affinity binding enabling a prolonged half-life compared with recombinant TRAIL, this specificity may be problematic when looking for a therapeutic effect as there are two active TRAIL receptors and it is not known which receptor is more important for apoptotic signalling. This is a potential explanation for the disappointing results with these agents in clinical trials. ${ }^{10} 11$ 
Bone marrow-derived mesenchymal stem cells (MSCs) are attractive candidates as vectors for anticancer therapies for multiple reasons. In vitro migration studies have demonstrated MSC migration towards both tumour cells and their conditioned media $^{12} 13$ while in vivo MSCs have been shown to incorporate into and persist in tumours following systemic administration in a wide variety of tumour models, including lung metastases ${ }^{14}$ and glioma. ${ }^{15}$ Multiple delivery routes are also effective for MSC therapy, including intravenous, direct intramyocardial ${ }^{16}$ and intraperitoneal delivery. ${ }^{17}$ While multiple growth factors and chemokines have been postulated to be important in MSC homing, the precise mechanism by which MSCs accumulate within tumours is poorly understood. ${ }^{18}$

In this study, we show for the first time that MSCs expressing TRAIL (MSCTRAIL) induce apoptosis in MPM cells in vitro and that MSCs home to and incorporate into tumours in vivo when delivered via both intrapleural and intravenous routes. Furthermore, we demonstrate that intravenous MSCTRAIL delivery causes a significant reduction in tumour growth in an in vivo model of MPM through a mechanism involving increased intratumoural MSC retention and tumour cell apoptosis.

\section{MATERIALS AND METHODS}

\section{Cell culture}

Tissue culture reagents were purchased from Invitrogen (Paisley, UK) unless otherwise stated. Human adult bone marrow-derived MSCs were purchased from Tulane University and cultured in $\alpha$-MEM with $16 \%$ fetal bovine serum (FBS), 4 mM L-glutamine with $50 \mathrm{U} / \mathrm{mL}$ penicillin and $50 \mu \mathrm{g} / \mathrm{mL}$ streptomycin. MSCs transduced with a Tet-inducible plasmid had FBS replaced with Tet-system approved FBS (Clontech, Paris, France). Human MPM cell lines (MSTO-211H, H28, H2052, ONE58, JU77 and LO68) and the benign mesothelial cell line Met5A were a kind gift from Professor Bruce Robinson (University of Western Australia) and were cultured in Dulbecco's Modified Eagle Medium with 10\% FBS, $4 \mathrm{mM}$ L-glutamine with $50 \mathrm{U} / \mathrm{mL}$ penicillin and $50 \mu \mathrm{g} / \mathrm{mL}$ streptomycin.

\section{Lentiviral vectors}

TRAIL-IRES-eGFP lentivirus vector was produced as previously described. ${ }^{14}$ Luciferase plasmid, pLIONII-HYG-Luc2YFP, was a gift from Dr Stephen Goldie (Cancer Research Institute, Cambridge), and lentivirus was produced using calcium phosphate transfection as described. ${ }^{19}$

MSCs were transduced with the TRAIL-IRES-eGFP lentivirus as previously described. ${ }^{14}$ Human TRAIL protein production with and without TRAIL activation in both cell supernatant and lysates was confirmed by ELISA (R\&D Systems). MSTO-211H and $\mathrm{H} 28$ cells were transduced with pLIONII-HYG-Luc2YFP and selected with hygromycin $200 \mu \mathrm{g} / \mathrm{mL}$ until a pure population was achieved.

\section{Flow cytometry for TRAIL receptors}

MPM cells were harvested and resuspended at $1 \times 10^{6}$ cells $/ \mathrm{mL}$, then incubated with antibodies against the four TRAIL receptors or an isotype control, followed by biotinylated secondary goat antimouse IgG1 and streptavidin phycoerythrin (PE). Flow cytometry was performed to detect PE.

\section{In vitro co-culture experiments}

MSCTRAIL cells were plated in a 1:1 ratio with human MPM cells, and apoptosis and cell death were determined as described in online supplementary materials and methods.
In vivo pleural mesothelioma model

All animal studies were approved by the University College London Biological Services Ethical Review Committee and licensed under the UK Home Office regulations and the Evidence for the Operation of Animals (Scientific Procedures) Act 1986 (Home Office, London, UK). Eight-week-old female NOD/SCID mice were purchased from Harlan, kept in individually ventilated cages under specific pathogen-free conditions and had access to sterile-irradiated food and autoclaved water ad libitum.

To create pleural tumours, mice were anaesthetised using 2\% isofluorane, the right thoracic wall was shaved and cleaned with alcohol. A $5 \mathrm{~mm}$ incision was made on the right chest wall, and a right anterolateral thoracotomy was performed in the fourth intercostal space, followed by installation of luciferasetransduced MSTO-211H (MSTO-211HLuc) cells in $100 \mu \mathrm{L}$ phosphate-buffered saline (PBS). MSCs were either delivered intrapleurally as described or intravenously using the lateral tail vein. Animals were weighed twice weekly, and bioluminescent imaging of tumour burden was performed twice weekly. Tumours were allowed to develop until mice reached 20\% weight loss or showed signs of distress.

\section{In vivo imaging of MSC homing}

$8 \times 10^{4}$ MSTO-211HLuc were delivered intrapleurally and tumours were left to develop for 5 days. $1 \times 10^{6}$ untransduced MSCs were labelled using the fluorescent lipophilic dyes, DiI (1,1'-dioctadecyl-3,3,3'3'-tetramethylindocarbocyanine perchlorate) and $\operatorname{DiR}\left(3,3,3^{\prime}, 3^{\prime}\right.$ tetramethylindotricarbocyanine iodide) according to manufacturer's instructions and delivered either by intravenous or intrapleural injections 5 days post-tumour cell injection. Bioluminescence and fluorescence were determined using an in vivo imaging system (IVIS Lumina, Caliper Life Sciences) as described in online supplementary methods. At study termination, mice were killed and tumour samples were digested for flow cytometry and fixed for histochemical analysis.

\section{Flow cytometry analysis of tumours}

Tumours were identified using open cavity bioluminescent imaging, removed and digested in a solution containing RPMI-1640 (Invitrogen) with $1 \mathrm{mg} / \mathrm{mL}$ collagenase (Sigma) and DNase I (Roche; $10 \mu \mathrm{g} / \mathrm{mL}$ ) for $1 \mathrm{~h}$ at $37^{\circ} \mathrm{C}$. Red blood cells were lysed with red blood cell lysis buffer (Sigma) for $1 \mathrm{~min}$, neutralised with RPMI and filtered. Flow cytometry (LSR Fortessa, Beckton Dickinson) was performed for YFP to detect tumour cells and DiR to detect MSCs.

\section{In vivo therapeutic effect of MSCTRAIL}

Pleural tumours were established as previously described. Tumours were left to develop for 5 days and for intravenous delivery $1 \times 10^{6}$ MSCTRAIL or untransduced MSCs suspended in PBS or PBS alone were delivered intravenously on days 5, 9, 12, 15 and 18. For intrapleural delivery, the same protocol was followed except that animals received intrapleurally delivered MSCTRAIL, untransduced MSCs or PBS alone on days 5, 9, 12, 15 and 18. All animals received doxycycline at $2 \mathrm{mg} / \mathrm{mL}$ in sterile water containing 3\% sucrose. Tumour growth was monitored via bioluminescent imaging twice weekly using IVIS and tumour burden was determined as described above. Animals were injected with $10 \mathrm{mg} / \mathrm{kg} \mathrm{5}$-bromo-2'-deoxyuridine (BrdU; Invitrogen) $1 \mathrm{~h}$ prior to sacrifice. All tumour tissue and lungs were removed and weighed prior to fixation and fixed overnight in $10 \%$ neutral buffered formalin (Roche) for histology. 


\section{Immunohistochemistry}

Samples were processed as described in online supplementary methods. Calretinin antibody (Abcam), WT1 antibody (Upstate Cell Signaling Solutions, New York, USA), TRAIL, DR5 antibodies (ProSci) and Luciferase antibodies (rabbit polyclonal; Abcam) were used as primary antibodies. BrdU-positive and TUNEL-positive cells were quantified using Volocity software.

\section{Statistics}

Statistical analysis was performed using GraphPad Prism V.4 (GraphPad Software). In vivo experiments with multiple groups were analysed using repeated measures ANOVA, and singlegroup data were assessed using Student $t$ test. All in vitro experiments were performed in triplicate unless specified.

\section{RESULTS}

\section{Characterisation of cells and transduction of MSCs}

The ability of MSCs to differentiate into fat and bone was confirmed along with their colony-forming efficiency as previously described. ${ }^{14}$ MSC transduction with TRAIL-IRES-eGFP under the control of a tetracycline-dependent promoter was successful (figure 1A,B), and transduction efficiency was $>96 \%$ following activation with doxycycline (figure 1C). ELISA confirmed high TRAIL expression in MSC cell lysates following TRAIL activation with doxycycline but low levels in cell lysates from inactivated MSCs and cell supernatants (figure 1D).

Immunocytochemistry confirmed both calretinin and Wilms Tumour antigen 1 (WT1) in all MPM cell lines (see online supplementary figure $\mathrm{S} 1 \mathrm{~A}-\mathrm{F}$ ), and they all possessed death receptor 5 (DR5) (see online supplementary figure S1G), the receptor responsible for the majority of TRAIL signalling. There was no correlation between the mean fluorescence intensity of DR5 staining and the sensitivity of MPM to MSCTRAIL (see online supplementary figure $\mathrm{S} 1 \mathrm{H}$ ). Met5A is shown as a normal mesothelial control.

\section{In vitro co-culture experiments demonstrate variable sensitivity of MPM cells to MSCTRAIL}

MPM tumour cell lines were co-cultured for $48 \mathrm{~h}$ with rTRAIL or MSCTRAIL with doxycycline to activate TRAIL production. Five out of six cell lines (83\%) showed sensitivity to TRAIL. H28 cells showed a significant increase in apoptosis and death when treated with MSCTRAIL compared with either rTRAIL $(3.6 \pm 0.1 \%$ vs $11.3 \pm 0.7 \%, \quad \mathrm{p}=0.007) \quad$ or inactivated MSCTRAIL $(3.4 \pm 0.1 \%$ vs $11.3 \pm 0.7 \%, \mathrm{p}=0.001$ ) (figure $2 \mathrm{~A}$, D). MSTO-211H cells were sensitive to treatment with rTRAIL showing over $40 \%$ cell death compared with inactivated MSCTRAIL (42.6 $\pm 4.2 \%$ vs $12.9 \pm 0.8 \%, \mathrm{p}<0.0001)$, which increased to over $58 \%$ when treated with MSCTRAIL (12.9 $\pm 0.8 \%$ vs $58.2 \pm 1.2 \%, \mathrm{p}<0.0001$ ) (figure $2 \mathrm{~B}, \mathrm{D}$ ). In Met5A, there was no significant increase in apoptosis and death when treated with either rTRAIL or MSCTRAIL compared with inactivated MSCTRAIL $(1.5 \pm 0.2 \%$ and $1.9 \pm 1.1 \%$, respectively, vs $0.8 \pm 0.05 \%, p=0.283$ and $\mathrm{p}=0.285$, respectively) (figure $2 \mathrm{C}$, D). ONE58, JU77, H2052 and LO68 were also tested and were sensitive to MSCTRAIL (see online supplementary Fig S2A).

To confirm that cell death was not caused by treatment with doxycycline, MPM cells were incubated for $48 \mathrm{~h}$ with $10 \mu \mathrm{g} / \mathrm{mL}$ doxycycline, harvested and stained for annexin V and DAPI as before. Flow cytometry showed no significant increase in cell death or apoptosis in any of the MPM cell lines tested (see online supplementary figure S2B).
Bioluminescence is suitable for monitoring longitudinal MPM tumour growth and a quantitative end point of tumour burden

MSTO-211H cells were transduced with a luciferase-YFP (MSTO-211HLuc) lentiviral vector and selected using hygromycin $(200 \mu \mathrm{g} / \mathrm{mL})$ to ensure a pure population (figure $3 \mathrm{~A}$ ) and increasing cancer cell numbers correlated well with increasing bioluminescent signal (figure 3B,C).

To determine the kinetics of tumour growth in vivo, $8 \times 10^{4}$ MSTO-211HLuc cells were injected intrapleurally and tumour growth was monitored. Bioluminescence images showed increasing signal over 21 days (figure 3D,E). MSTO-211H cells were used as they are known to be tumourigenic in vivo while $\mathrm{H} 28$ are not. ${ }^{20}$

\section{Intrapleurally and intravenously delivered MSCs home to MPM in vivo}

Tumours were established as described in the methods with $1 \times 10^{6}$ DiI-labelled MSCs injected into mice either intrapleurally or intravenously. Animals were imaged immediately after injection and $48 \mathrm{~h}$ later to determine the location of both MSCs and tumours. Tumours were clearly visible on bioluminescent imaging (figure 4A-C), and fluorescence demonstrated MSC localisation at the site of the tumours (figure 4E,F). No animals developed pleural effusions so signal is representative of solid tumours. Control animals receiving no MSCs showed no fluorescent signal, confirming that any signal detected was representative of MSCs and not luciferase-YFP (figure 4D). Immunofluorescence confirmed that DiI-labelled MSCs were located within the tumour stroma when delivered both intrapleurally and intravenously (figure 4G-J). No fluorescent signal was seen outside the lungs, suggesting that MSCs did not reach other organs and there was no evidence of toxicity.

\section{MSCTRAIL causes a reduction in tumour growth when delivered intravenously but not intrapleurally}

Tumours were established as described and $1 \times 10^{6}$ MSCTRAIL or untransduced MSC or $100 \mu \mathrm{L}$ PBS were delivered intravenously on days $5,9,12,15$ and 18. IVIS imaging demonstrated a significant reduction in tumour growth in the MSCTRAIL group compared with the PBS and untransduced MSC group $(\mathrm{p}<0.001$, repeated measures ANOVA; figure 5A,B). There was also a significant reduction in lung weights in MSCTRAIL-treated mice $(\mathrm{p}<0.0391$; figure 5C).

To determine whether a similar effect was seen with topically delivered MSCTRAIL, the experiment was repeated using intrapleural delivery of $1 \times 10^{6}$ MSCTRAIL or untransduced MSC or PBS. In this experiment, there was no significant reduction in tumour growth or lung weights compared with the PBS and untransduced MSC-treated groups.

Histopathological analysis showed no significant difference in tumour cell proliferation (figure 5D-F and $\mathrm{K}$ ), but there was a significant increase in apoptosis in tumours from the intravenous MSCTRAIL-treated group ( $\mathrm{p}<0.0053$; figures 5G-J and L), suggesting that intravenous MSCTRAIL cells reduce tumour size by inducing apoptosis. Representative H\&E sections from tumours treated with PBS, intrapleural MSCTRAIL and intravenous MSCTRAIL (see online supplementary figure S3) show the presence of pyknotic nuclei and dead cells in the intravenous MSCTRAIL-treated tumour. 

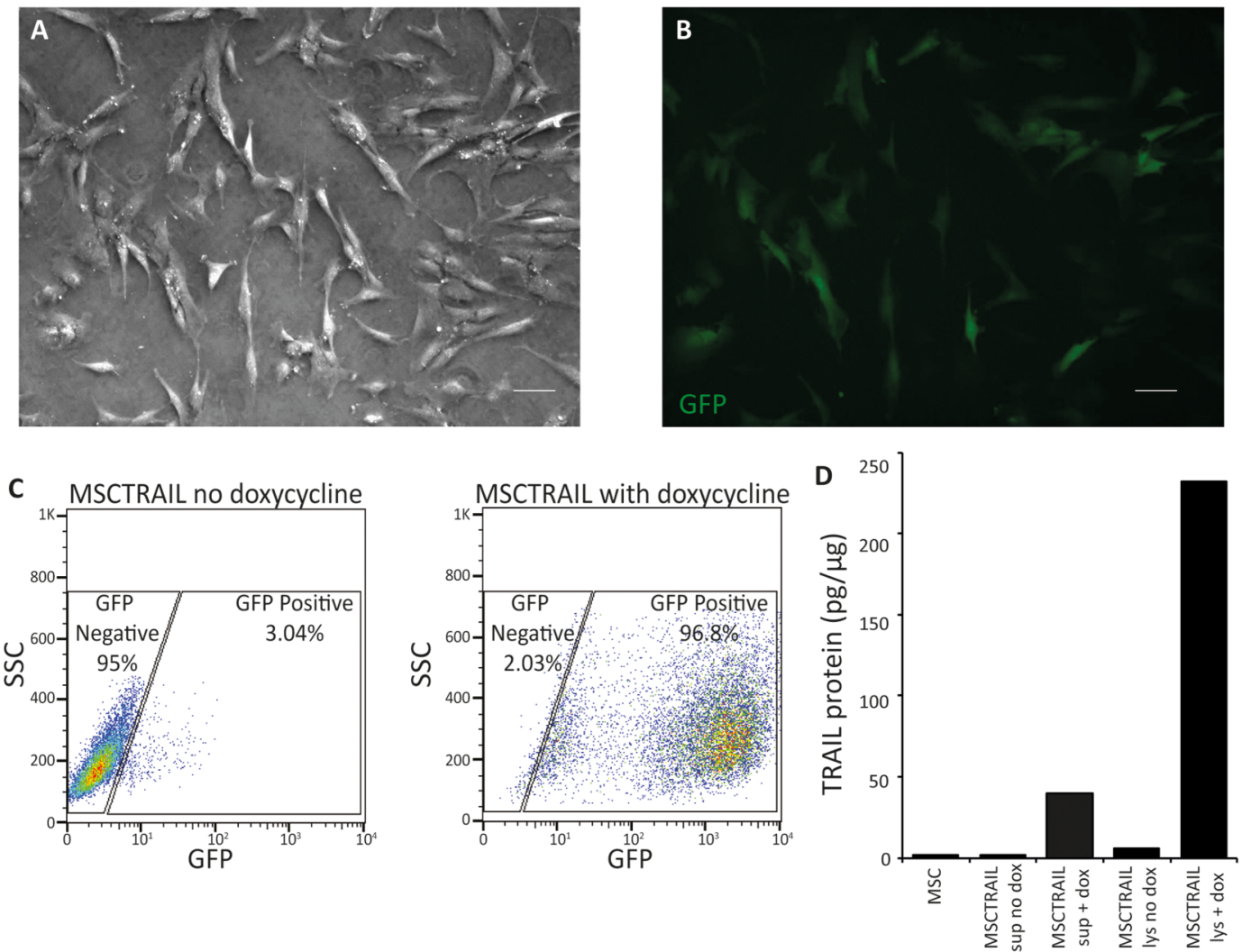

Figure 1 MSC transduction. (A) Bright field and (B) fluorescence microscopy to confirm GFP expression following transduction of MSC with TRAIL-IRES-eGFP lentivirus and activation with doxycycline $(10 \mu \mathrm{g} / \mathrm{mL}$ ) (magnification, $\times 5$; bar $20 \mu \mathrm{m})$. (C) Flow cytometry plots confirming efficiency of MSC transduction following TRAIL activation with doxycycline and (D) a TRAIL ELISA of MSC cell supernatant and lysate demonstrating the production of TRAIL protein in cell lysates following MSCTRAIL activation with doxycycline. There is minimal TRAIL production following MSC transduction in the absence of doxycycline and low levels in cell supernatant from activated MSCTRAIL. MSC, mesenchymal stem cell; MSCTRAIL, MSCs expressing TRAIL; TRAIL, tumour necrosis factor-related apoptosis-inducing ligand.

Intravenous-delivered MSCs incorporate into tumours in greater numbers than intrapleural- delivered MSCs

To determine why MSCTRAIL is only effective when delivered intravenously, mice were given $2 \times 10^{5}$ MSTO-211HLuc intrapleurally and tumours were left to establish for 10 days. $1 \times 10^{6}$ DiR-stained MSCs stained were injected either intravenously or intrapleurally and imaging was performed daily. Tumours were successfully established and fluorescent MSCs were clearly visible following both routes of delivery (figure 6A). There was a significant difference in signal $24 \mathrm{~h}$ post-MSC injection $(\mathrm{p}=0.0125$; figure 6B), which was maintained throughout the imaging period, suggesting that MSCs incorporate into tumours in greater numbers when delivered intravenously compared with intrapleurally. Flow cytometry of tumour digests confirmed a greater percentage of MSCs in the tumours receiving intravenous MSCs (figure 6C,D), although the overall percentage was low compared with the number of tumour stromal cells and lung cells.

\section{DISCUSSION}

In this study, we have shown that MSCs engineered to express TRAIL can induce death in multiple MPM cell lines in vitro and are more efficient at killing than recombinant TRAIL. We have also demonstrated that MSCs migrate to MPM tumours in vivo when delivered both intravenously and intrapleurally. However, only intravenous-delivered MSCTRAIL causes a significant reduction in tumour growth and the difference in efficacy is likely due to a higher number of MSCs engrafting within the tumours causing increased apoptosis.
Using MSCs as vectors for gene therapy is becoming increasingly common as they are easy to extract from bone marrow, are highly expandable and readily transducible with viral vectors. ${ }^{21}$ Once modified they maintain their stem cell properties $^{22}$ and can be injected into a recipient without provoking an immune response. ${ }^{23}$ Clinical trials using allogeneic and autologous MSCs in cardiovascular and respiratory diseases have shown no adverse events or immunological reactions, ${ }^{24}{ }^{25}$ but as yet there are no clinical trials using engineered MSCs in cancer. The issue of immunological reactions will clearly need to be monitored for in any phase I clinical trial with engineered MSCs. MSCs home to multiple tumour types in vitro and in vivo, and while the precise mechanism of this has not been clearly established multiple cytokine/receptor pairs have been investigated. ${ }^{26}$ In our study, we show that MSCs home to MPM when delivered both intravenously and intrapleurally and are incorporated within the tumour tissue. These properties enable delivery of high-dose-targeted cancer therapy directly to the site of the tumour while reducing off-target effects, making it a clinically attractive option.

There is some concern that exogenously delivered MSCs have an unpredictable effect on tumour biology with different in vivo models suggesting pro-tumourigenic or antitumourigenic properties, ${ }^{27}$ but MSCs genetically modified to express pro-apoptotic molecules ensure an antitumourigenic effect. ${ }^{28}$ This is in line with our results where MSCs alone had no pro-tumourigenic effect and intravenous MSCTRAIL had an antitumourigenic effect. TRAIL is an exciting prospect for cancer therapy because 

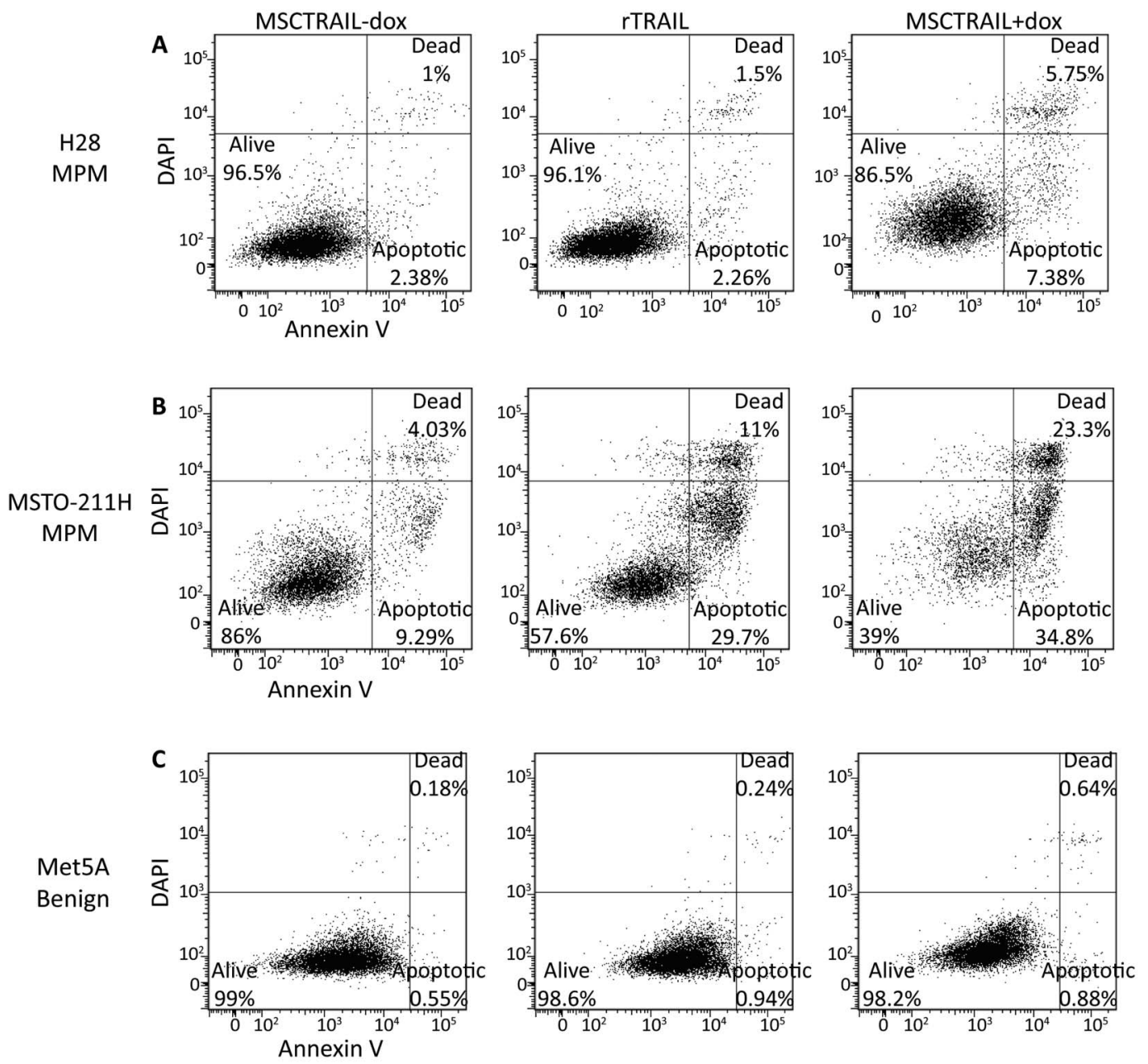

D

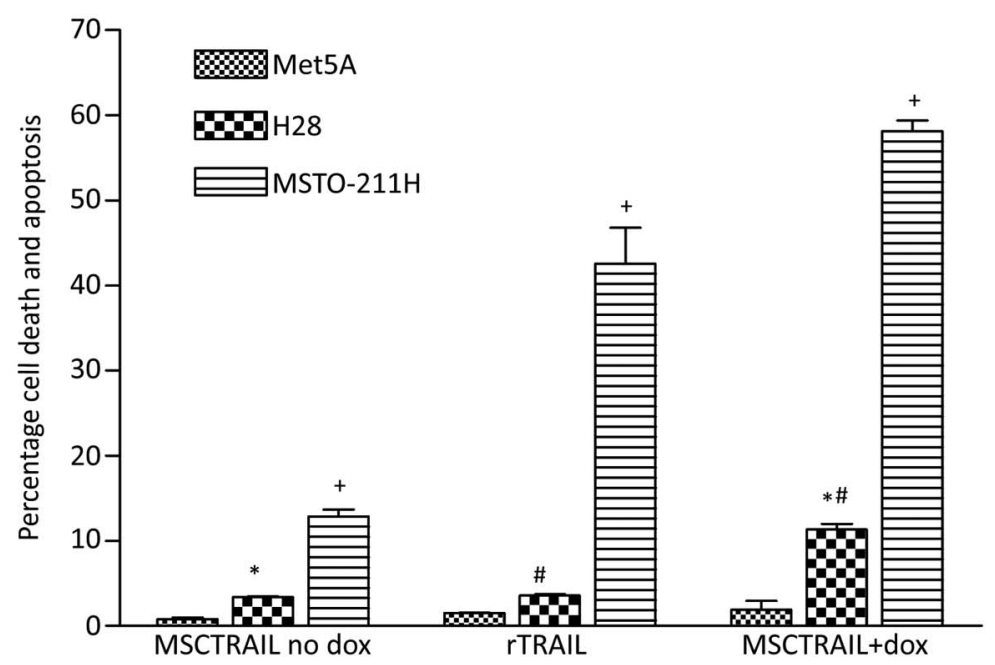

Figure 2 Human MPM exhibit variable in vitro sensitivity to rTRAIL and MSCTRAIL. Flow cytometry plots showing increased apoptosis and cell death in (A) H28 MPM when treated with rTRAIL and MSCTRAIL+dox and (B) in MSTO-211H MPM cell line with MSCTRAIL+dox compared with rTRAIL or MSCTRAIL no dox. Control benign mesothelial cells Met5A, (C) show no significant apoptosis or cell death when treated with rTRAIL or MSCTRAIL+dox. Apoptosis and death were determined using annexin V and DAPI staining, respectively. (D) Flow cytometry data from co-culture experiments showing an increase in apoptosis and death in both $\mathrm{H} 28$ and MSTO-211H following treatment with rTRAIL and MSCTRAIL. (+p<0.0001; $\left.{ }^{*} \mathrm{p}=0.001 ; \# p=0.007\right)$. MPM, malignant pleural mesothelioma; MSCTRAIL, mesenchymal stem cells expressing TRAIL; TRAIL, tumour necrosis factor-related apoptosis-inducing ligand.

of its ability to selectively target cancer cells without killing healthy cells; however, its short half-life ${ }^{6}$ means repeated highdose systemic treatment would be required to produce a significant local effect. While human MPM cell lines express both DR4 and DR5 receptors with higher levels of DR5 than DR4, this expression does not correlate with TRAIL 
A

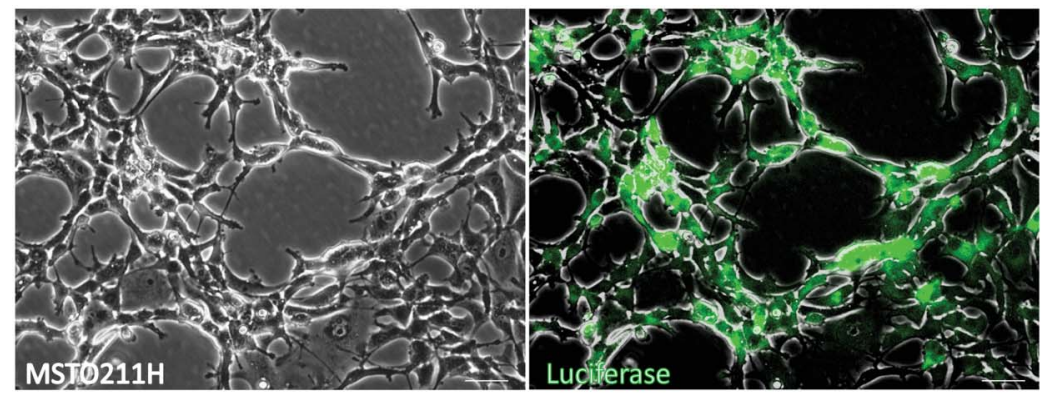

B
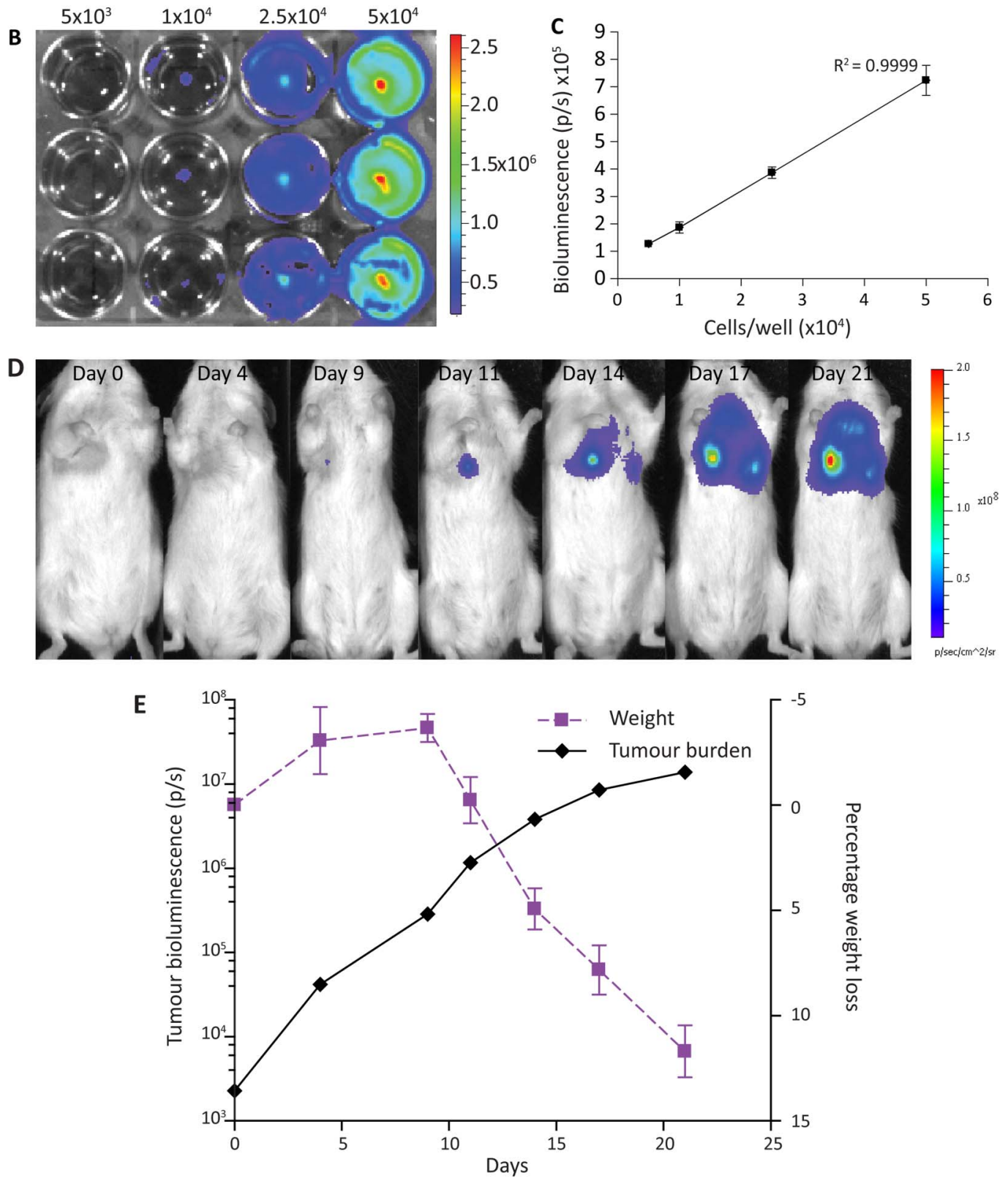

Figure 3 Luciferase-transduced MSTO-211H cells injected intrapleurally can be tracked longitudinally and correspond to tumour growth. (A) Human MPM cells MSTO-211H were transduced with pLIONII-HYG-Luc2YFP and selected with hygromycin $(200 \mu \mathrm{g} / \mathrm{mL})$ until a pure population was achieved (phase contrast, left panel and immunofluorescence of luciferase transduced, right panel) (magnification $5 \times$; bar $60 \mu \mathrm{m}$ ). (B) MST0211HLuc were plated in increasing numbers in a 12-well plate and imaging was performed once cells were adherent. (C) Graph of bioluminescent signal with increasing cell number showing good correlation between increasing signal and increasing cell number (represented as average \pm SEM). (D) IVIS images demonstrate that intrapleural delivery of MSTO-211HLuc cells results in an increase bioluminescent signal over time. (E) The most rapid increase in tumour growth occurs within the first 10 days following tumour cell inoculation. Weight loss does not occur until tumour growth slows, suggesting it is a late marker of disease. MPM, malignant pleural mesothelioma. 

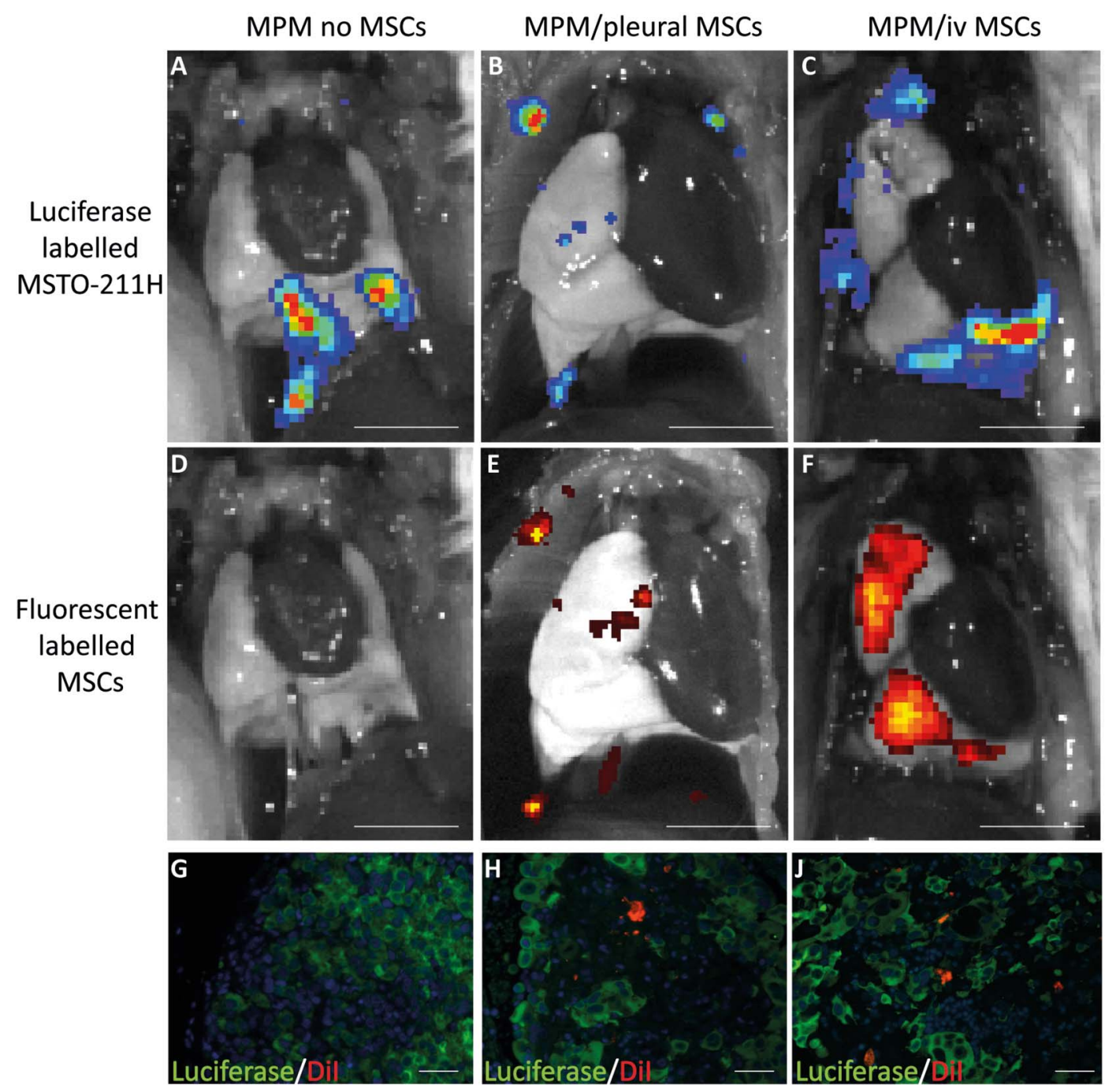

Figure 4 Human MSCs home to an in vivo model of MPM when delivered both intrapleurally and intravenously. (A-C) IVIS images of animals with intrapleural bioluminescent MST0-211HLuc tumour cells. (D) Lack of fluorescent signal in control animal with no DiR-labelled MSCs while animals receiving (E) pleural MSCs and (F) intravenous MSCs show co-localisation of fluorescent MSCs at the sites of tumour (scale bar 5 mm). (G) Immunofluorescence images confirm the absence of Dil-labelled MSCs (red) within luciferase positive tumour, while MSCs are visible within the luciferase-positive tumours following both $(\mathrm{H})$ intrapleural and $(\mathrm{J})$ intravenous MSC delivery (magnification $\times 20 ;$ bar $60 \mu \mathrm{m}$ ). MPM, malignant pleural mesothelioma; MSC, mesenchymal stem cell.

sensitivity. ${ }^{29}$ There are currently no biomarkers to predict sensitivity to TRAIL. The efficacy of rTRAIL-mediated killing of MPM has been assessed in vitro in combination with multiple other therapies, ${ }^{30}{ }^{31}$ but not as a single agent or in vivo models. MSCTRAIL is known to eliminate or reduce tumour growth in other in vivo cancer models, ${ }^{14} 1532$ but has not been assessed in MPM. It can kill the cancer stem cell-like populations of cancer cells that are thought to be resistant to current chemotherapies and are a postulated mechanism for tumour recurrence following treatment ${ }^{33}$. Our results demonstrate for the first time that MPM cells are sensitive to treatment with MSCTRAIL and there is a greater level of cell death compared with treatment with rTRAIL in vitro. This treatment remains effective in vivo with a significant reduction in tumour burden when MSCTRAIL is delivered intravenously. During our experiments, the number of MSCTRAIL cells injected appears high relative to tumour cell inoculation. However, when the population doubling time of the MSTO-211H cells of $20 \mathrm{~h}$ is considered, the ratio of tumour cells to MSCTRAIL cells would be significantly lower. ${ }^{34}$
When using MSCs as therapeutic delivery vectors, treatment efficacy is related to the level of MSC accumulation at tumour sites. Our study shows that while MSC homing occurs regardless of route of delivery, the level of MSC accumulation following intravenous delivery is significantly higher than when delivered intrapleurally. The increase in apoptosis seen in the intravenous delivery model could be a direct effect of the tumour receiving a greater number of cells and hence a higher dose of TRAIL. The first step in MSC accumulation within tumours is adhesion to vascular endothelial cells, and multiple factors are involved in this process. ${ }^{35} 36$ It may be that cells delivered intravenously have higher accumulation as they are delivered directly to endothelial cells, making adhesion more likely. Alternatively intrapleurally delivered cells may have lower intratumoural accumulation as their delivery is likely to be to areas that are poorly vascularised and hypoxic and MSCs are more likely to die when located within a hypoxic microenvironment. ${ }^{37}$

In conclusion, this study shows intravenous MSCTRAIL delivery causes a reduction in tumour growth in an in vivo MPM model. MSCs home to and incorporate into tumours using both 


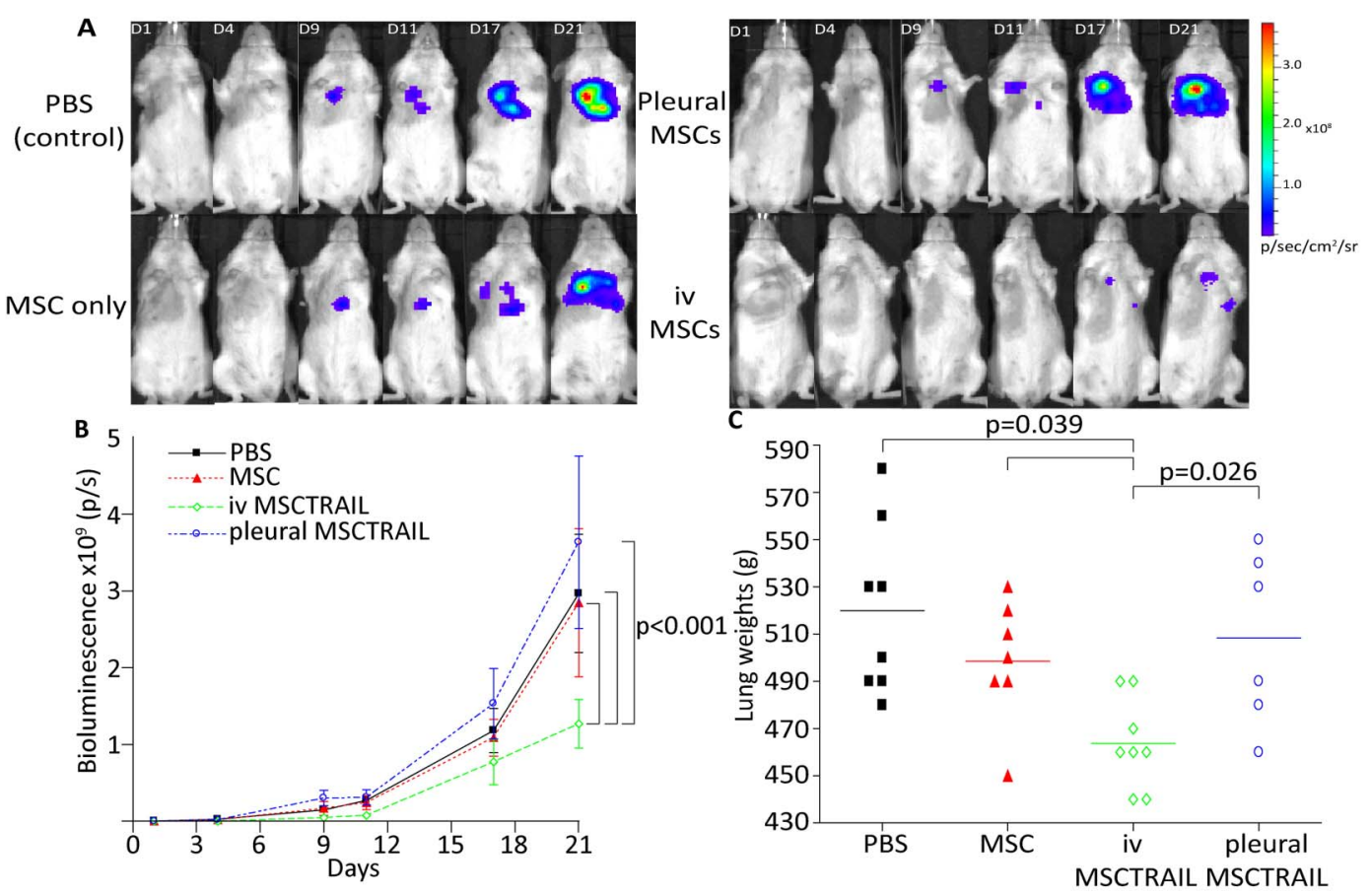

Figure 5 MSCTRAIL reduce the growth of MPM when delivered intravenously. (A) IVIS images of representative animals from each experimental group showing reduced bioluminescent signal in animals treated with intravenous MSCTRAIL. (B) Line graph to demonstrate a reduction in total photon count $(\mathrm{p} / \mathrm{s})$ seen in animals in the intravenous MSCTRAIL-treated group over 21 days compared with MSC delivery alone, PBS or intrapleural MSCTRAIL. Photon count was determined using dedicated regions of interest around the whole body to include both pleural tumour burden and any distant metastatic deposits (Living Image Software, Caliper LifeSciences; $p<0.001$ ). (C) Dot plot to show a reduction in lung weights with intravenous MSCTRAIL treatment compared with all other treatment groups $(p<0.05)$. Immunofluorescence showing proliferating cells $($ red) and luciferase-positive tumour cells (green) in tumours treated with (D) PBS, (E) intrapleural-delivered MSCTRAIL and (F) intravenous-delivered MSCTRAIL. (G) Representative immunofluorescence showing TUNEL-positive apoptotic cells (green) within pleural tumours (red) in animals treated with (G) PBS, (H) pleural MSCTRAIL and (J) increased apoptosis in tumours treated with intravenous MSCTRAIL (magnification $4 \times$, bar $60 \mu \mathrm{m}$ ). (K) Proliferating cells per tumour area were quantified (Volocity Software) with no significant difference in the number of BrdU-positive cells between the treatment groups. (L) TUNEL-positive cells per tumour area were quantified (Volocity Software), and MSCTRAIL-treated animals showed increased levels of apoptosis within tumours compared with all other groups ( $p=0.0053)$. MPM, malignant pleural mesothelioma; MSC, mesenchymal stem cell; MSCTRAIL, MSCs expressing TRAIL; PBS, phosphate-buffered saline; TRAIL, tumour necrosis factor-related apoptosis-inducing ligand. 


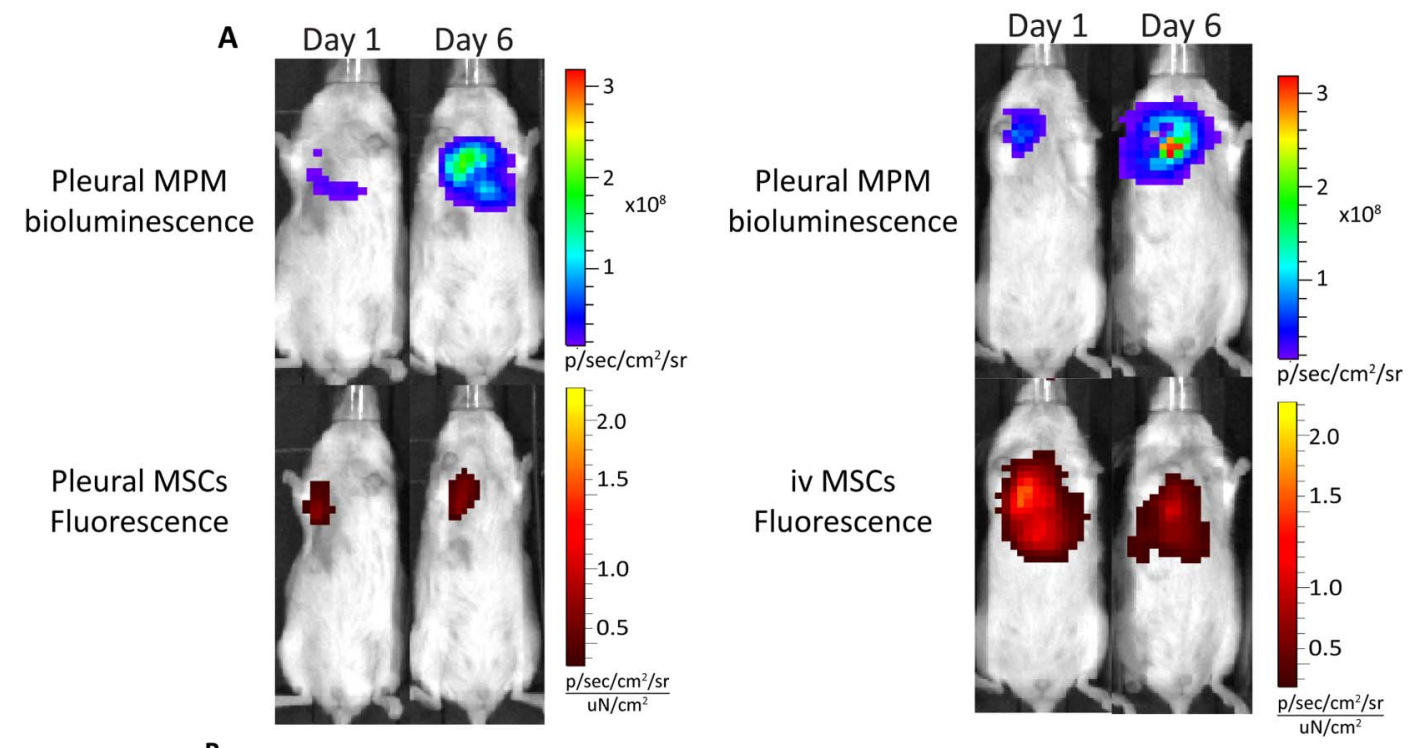

B

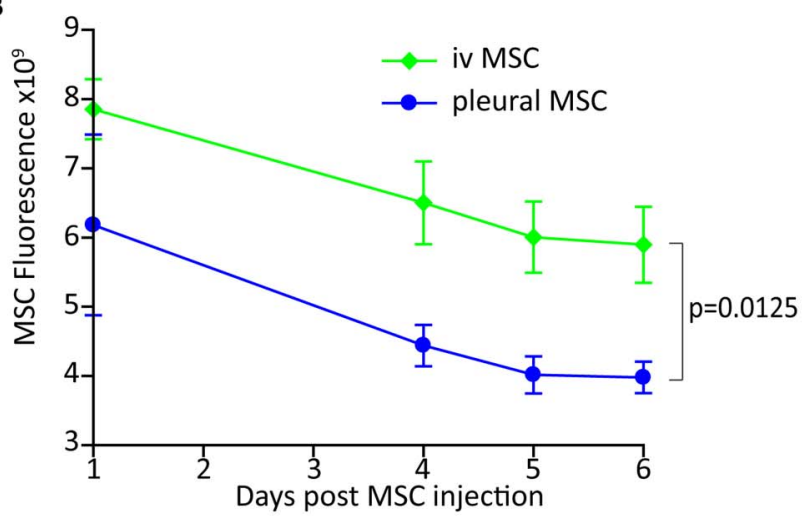

C
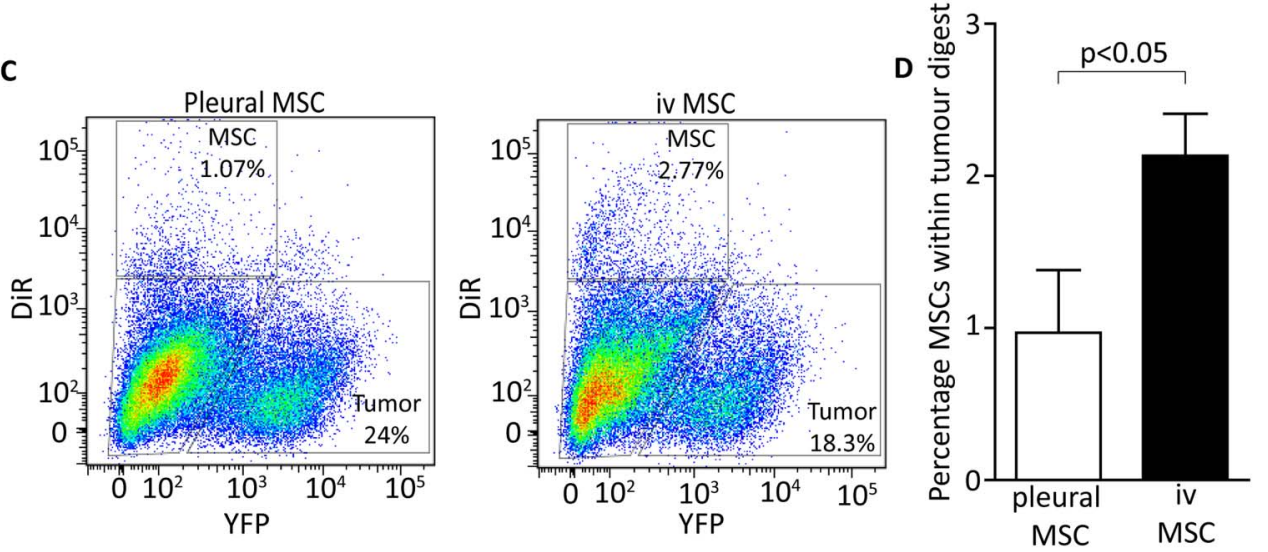

Figure 6 Intravenous-delivered MSCS are incorporated into tumours in greater numbers than when delivered intrapleurally. (A) IVIS images to show established bioluminescent MPM tumours and corresponding fluorescence from DiR-labelled MSCs on days 1 and 6 following MSC injection. Intravenous-delivered MSCs show a higher fluorescent signal on day 1 and day 6 following injection compared with intrapleural-delivered MSCs. (B) Fluorescent signal was quantified and MSCs delivered intravenously showed a higher signal on day 1 compared with cells delivered intrapleurally, which persisted until day $6(p=0.0125)$. (C) Tumours were removed and digested for flow cytometry, which revealed a higher percentage of DiR-stained MSCs in tumours given intravenous MSCs than in those given intrapleural MSCs. (D) Bar chart to show a significant increase in DiR-stained MSCs in tumour digests following intravempis delivery compared with pleural delivery $(p<0.0412)$. MPM, malignant pleural mesothelioma; MSC, mesenchymal stem cell.

intravenous and intrapleural delivery routes but greater numbers engraft when delivered intravenously. The therapeutic effect seen with intravenous delivery could be related to greater engraftment of MSCs within the tumour and is an important finding when considering the future therapeutic role of MSCTRAIL therapy in the clinic.
Contributors EKS, AG and SMJ designed research and analysed data. EKS, AG, KKK and KMN performed research. DD, SDSL, TLK and KLO provided technical assistance. GYCL contributed cell lines and reagents. EKS and SMJ wrote the manuscript.

Funding MRC, British Lung Foundation, Mick Knighton Mesothelioma Research Fund (G1000355, EKS). European Research Council Starting Grant (260290, AG). Wellcome Trust Senior Clinical Fellowship (WT091730AIA, SMJ). Department of Health-NIHR Biomedical Research Centre (AG, SMJ). 


\section{Competing interests None.}

Provenance and peer review Not commissioned; externally peer reviewed.

Open Access This is an Open Access article distributed in accordance with the terms of the Creative Commons Attribution (CC BY 3.0) license, which permits others to distribute, remix, adapt and build upon this work, for commercial use, provided the original work is properly cited. See: http://creativecommons.org/licenses/ by/3.0/

\section{REFERENCES}

1 Wagner JC, Sleggs CA, Marchand P. Diffuse pleural mesothelioma and asbestos exposure in the North Western Cape Province. Br J Ind Med 1960;17:260-71.

2 Executive HaS. Mesothelioma Register. http://wwwhsegov/statistics/causdis/ mesothelioma/. 2010. http://www.hse.gov/statistics/causdis/mesothelioma/http:// www.hse.gov/statistics/causdis/mesothelioma/):http://www.hse.gov/statistics/causdis/ mesothelioma/. Epub http://www.hse.gov/statistics/causdis/mesothelioma/. http:/l www.hse.gov/statistics/causdis/mesothelioma/

3 Vogelzang NJ, Rusthoven JJ, Symanowski J, et al. Phase III study of pemetrexed in combination with cisplatin versus cisplatin alone in patients with malignant pleural mesothelioma. J Clin Oncol 2003;21:2636-44.

4 Treasure T, Tan C, Lang-Lazdunski L, et al. The MARS trial: mesothelioma and radical surgery. Interact Cardiovasc Thorac Surg 2006:5:58-9.

5 Fennell DA, Rudd RM. Defective core-apoptosis signalling in diffuse malignant pleural mesothelioma: opportunities for effective drug development. Lancet Oncol 2004;5:354-62.

6 Ashkenazi A, Pai RC, Fong S, et al. Safety and antitumor activity of recombinant soluble Apo2 ligand. J Clin Invest 1999;104:155-62.

7 Herbst RS, Eckhardt SG, Kurzrock R, et al. Phase I dose-escalation study of recombinant human Apo2L/TRAIL, a dual proapoptotic receptor agonist, in patients with advanced cancer. J Clin Oncol 2010;28:2839-46.

8 Hotte SJ, Hirte HW, Chen EX, et al. A phase 1 study of mapatumumab (fully human monoclonal antibody to TRAIL-R) in patients with advanced solid malignancies. (lin Cancer Res 2008:14:3450-5.

9 Plummer R, Attard G, Pacey S, et al. Phase 1 and pharmacokinetic study of lexatumumab in patients with advanced cancers. Clin Cancer Res 2007;13:6187-94.

10 Greco FA, Bonomi P, Crawford J, et al. Phase 2 study of mapatumumab, a fully human agonistic monoclonal antibody which targets and activates the TRAIL receptor-1, in patients with advanced non-small cell lung cancer. Lung Cancer 2008:61:82-90.

11 Camidge DR, Herbst RS, Gordon MS, et al. A phase I safety and pharmacokinetic study of the death receptor 5 agonistic antibody PR095780 in patients with advanced malignancies. Clin Cancer Res 2010;16:1256-63.

12 Menon LG, Picinich $\mathrm{S}$, Koneru $\mathrm{R}$, et al. Differential gene expression associated with migration of mesenchymal stem cells to conditioned medium from tumor cells or bone marrow cells. Stem Cells 2007:25:520-8.

13 Xin $\mathrm{H}$, Kanehira M, Mizuguchi $\mathrm{H}$, et al. Targeted delivery of CX3CL1 to multiple lung tumors by mesenchymal stem cells. Stem Cells 2007;25:1618-26.

14 Loebinger MR, Eddaoudi A, Davies D, et al. Mesenchymal stem cell delivery of TRAIL can eliminate metastatic cancer. Cancer Res 2009;69:4134-42.

15 Sasportas LS, Kasmieh R, Wakimoto H, et al. Assessment of therapeutic efficacy and fate of engineered human mesenchymal stem cells for cancer therapy. Proc Natl Acad Sci USA 2009;106:4822-7.

16 Mathiasen $A B$, Jorgensen $E$, Qayyum $A A$, et al. Rationale and design of the first randomized, double-blind, placebo-controlled trial of intramyocardial injection of autologous bone-marrow derived Mesenchymal Stromal Cells in chronic ischemic Heart Failure (MSC-HF Trial). Am Heart J 2012:164:285-91.
17 Kidd S, Spaeth E, Dembinski JL, et al. Direct evidence of mesenchymal stem cell tropism for tumor and wounding microenvironments using in vivo bioluminescent imaging. Stem Cells 2009:27:2614-23.

18 Loebinger MR, Janes SM. Stem cells as vectors for antitumour therapy. Thorax 2010;65:362-9.

19 Goldie SJ, Mulder KW, Tan DW, et al. FRMD4A upregulation in human squamous cell carcinoma promotes tumor growth and metastasis and is associated with poor prognosis. Cancer Res 2012;72:3424-36.

20 Veldwijk MR, Berlinghoff S, Jauch A, et al. Characterization of human mesothelioma cell lines as tumor models for suicide gene therapy. Onkologie 2008;31:91-6.

21 Lee K, Majumdar MK, Buyaner D, et al. Human mesenchymal stem cells maintain transgene expression during expansion and differentiation. Mol Ther 2001;3:857-66

22 Chan J, O'Donoghue $\mathrm{K}$, de la Fuente J, et al. Human fetal mesenchymal stem cells as vehicles for gene delivery. Stem Cells 2005;23:93-102.

23 Chen XC, Wang R, Zhao X, et al. Prophylaxis against carcinogenesis in three kinds of unestablished tumor models via IL12-gene-engineered MSCs. Carcinogenesis 2006;27:2434-41.

24 Hare JM, Fishman JE, Gerstenblith $\mathrm{G}$, et al. Comparison of allogeneic vs autologous bone marrow-derived mesenchymal stem cells delivered by transendocardial injection in patients with ischemic cardiomyopathy: the POSEIDON randomized trial. JAMA 2012;308:2369-79.

25 Weiss DJ, Casaburi R, Flannery $R$, et al. A placebo-controlled, randomized trial of mesenchymal stem cells in COPD. Chest 2013;143:1590-8.

26 Karp JM, Leng Teo GS. Mesenchymal stem cell homing: the devil is in the details. Cell Stem Cell 2009;4:206-16.

27 Klopp AH, Gupta A, Spaeth E, et al. Concise review: dissecting a discrepancy in the literature: do mesenchymal stem cells support or suppress tumor growth? Stem Cells 2011:29:11-19.

28 Millard SM, Fisk NM. Mesenchymal stem cells for systemic therapy: Shotgun approach or magic bullets? Bioessays 2013;35:173-82.

29 Belyanskaya LL, Marti TM, Hopkins-Donaldson S, et al. Human agonistic TRAll receptor antibodies Mapatumumab and Lexatumumab induce apoptosis in malignant mesothelioma and act synergistically with cisplatin. Mol Cancer 2007;6:66.

30 Liu W, Bodle E, Chen JY, et al. Tumor necrosis factor-related apoptosis-inducing ligand and chemotherapy cooperate to induce apoptosis in mesothelioma cell lines. Am J Respir Cell Mol Biol 2001;25:111-18.

31 Barbone D, Yang TM, Morgan JR, et al. Mammalian target of rapamycin contributes to the acquired apoptotic resistance of human mesothelioma multicellular spheroids. J Biol Chem 2008:283:13021-30.

32 Reagan MR, Seib FP, McMillin DW, et al. Stem cell implants for cancer therapy: TRAIL-expressing mesenchymal stem cells target cancer cells in situ. J Breast Cancer 2012;15:273-82

33 Loebinger MR, Sage EK, Davies D, et al. TRAll-expressing mesenchymal stem cells kill the putative cancer stem cell population. Br J Cancer 2010;103:1692-7.

34 Bepler G, Koehler A, Kiefer P, et al. Characterization of the state of differentiation of six newly established human non-small-cell lung cancer cell lines. Differentiation 1988;37:158-71

35 Uchibori R, Tsukahara T, Mizuguchi H, et al. NF-kappaB activity regulates mesenchymal stem cell accumulation at tumor sites. Cancer Res 2013;73:364-72.

36 Hosokawa Y, Hosokawa I, Ozaki K, et al. Cytokines differentially regulate ICAM-1 and VCAM-1 expression on human gingival fibroblasts. Clin Exp Immunol 2006:144:494-502.

37 Copland IB, Lord-Dufour S, Cuerquis J, et al. Improved autograft survival of mesenchymal stromal cells by plasminogen activator inhibitor 1 inhibition. Stem Cells 2009;27:467-77. 